\title{
Technical Note: A comparison of central and peripheral intraocular pressure using rebound tonometry
}

\author{
A. Queirós ${ }^{1}$, J. M. González-Méijome ${ }^{1}$, P. Fernandes ${ }^{1}$, J. Jorge ${ }^{1}$, \\ R. Montés-Micó ${ }^{2}$, J. B. Almeida ${ }^{1}$ and M. A. Parafita ${ }^{3}$ \\ ${ }^{1}$ Department of Physics (Optometry), School of Sciences, University of Minho, Braga, Portugal, \\ ${ }^{2}$ Department of Optics. University of Valencia, Valencia, Spain, and ${ }^{3}$ Department of Surgery \\ (Ophthalmology), School of Optics and Optometry, University of Santiago de Compostela, Spain
}

\begin{abstract}
Purpose: To compare central and peripheral intraocular pressure (IOP) readings obtained with rebound tonometry.

Methods: Intraocular pressure was measured on the right eye of 153 patients (65 males, 88 females), aged from 21 to 85 years (mean \pm S.D., $55.5 \pm 15.2$ years) with the ICare rebound tonometer at centre, and $2 \mathrm{~mm}$ from the limbus (in the nasal and temporal regions along the $0-180^{\circ}$ corneal meridian).

Results: Intraocular pressure values obtained with the ICare were $14.9 \pm 2.8 ; 14.1 \pm 2.5$ and $14.5 \pm 2.7 \mathrm{mmHg}$ at centre, nasal and temporal corneal locations, respectively. On average, nasal and temporal IOP readings were 0.75 and $0.37 \mathrm{mmHg}$ lower than the central reading $(p<0.05$ and $p>0.05$, respectively). A highly significant correlation was found between central and peripheral measurements in nasal $\left(r^{2}=0.905 ; p<0.001\right)$ and temporal $\left(r^{2}=0.879 ; p<0.001\right)$ regions along the horizontal meridian. Almost $80 \%$ of patients presented nasal IOP values within $\pm 1 \mathrm{mmHg}$ of the central value.

Conclusions: Intraocular pressure values measured with the ICare ${ }^{\circledR}$ rebound tonometer on the nasal corneal region is slightly lower on average and highly correlated with IOP values recorded at corneal centre. Both nasal and temporal readings are in good agreement with central IOP, and could be used to obtain a reliable estimate of rebound IOP in corneas where central readings cannot be taken.
\end{abstract}

Keywords: corneal structure' ICare ${ }^{\circledR}$, intraocular pressure, peripheral tonometry, rebound tonometry

\section{Introduction}

The assessment of intraocular pressure (IOP) is still of major importance in glaucoma diagnosis and follow-up. Enormous effort has been made in recent years to develop non-invasive, rapid and accurate methods to

Received: 6 December 2006

Revised form: 3 February 2007, 25 May 2007

Accepted: 14 June 2007

Correspondence and reprint requests to: José Manuel González-Méijome, Departamento de Física (Optometria), Campus de Gualtar, Universidade do Minho, 4710-057 Braga - Portugal.

Tel.: +351 25360 4067; Fax : +351 253678981 .

E-mail address: jgmeijome@fisica.uminho.pt measure IOP. Non-contact tonometers have been at the forefront of these efforts (Queiros et al., 2006), with the newest generation of devices showing high agreement with Goldmann applanation tonometry (GAT) in healthy (Jorge et al., 2002) and glaucomatous patients (Jorge et al., 2003).

The ICare ${ }^{\circledR}$ (Tiolat Oy, Helsinki, Finland) is a new portable tonometer that measures the IOP by processing the rebound movement of a rod probe resulting from its interaction with the eye. Experimental studies have been carried out by Kontiola and co-workers to calibrate the instrument (Kontiola, 2000), and its accuracy has been checked in rats (Kontiola et al., 2001) before proceeding with human clinical applications (Kontiola and Puska, 2004). More recently, this instrument has been 
compared with other clinical tonometers, providing rapid IOP measurements in good agreement with GAT (Fernandes et al., 2005; Davies et al., 2006; Iliev et al., 2006) and different portable tonometers (van der Jagt and Jansonius, 2005; Garcia-Resua et al., 2006) in healthy populations and also in patients with glaucoma and ocular hypertension (Martinez-de-la-Casa et al., 2005). There is a general agreement in the literature regarding a slight overestimation of IOP with ICare compared with GAT in its conventional (Fernandes et al., 2005; Martinez-de-la-Casa et al., 2005) and portable (Garcia-Resua et al., 2006) versions. Comparisons of ICare with dynamic contour tonometer also displayed an excellent agreement (Martinez-de-la-Casa et al., 2006b). A description of this instrument and its function can be obtained from the manufacturer's information and in recent publications by the inventors, and the authors of the present and other studies (Fernandes et al., 2005; Garcia-Resua et al., 2006).

ICare was primarily designed to fit to the small scale of the rat and mouse eye. As a consequence, an additional benefit of the ICare is the possibility of taking measurements at different corneal locations easily, using only a small part of the cornea. This could be of great clinical interest when reliable measurements could not be taken from the central part of the cornea using applanation tonometers because of the presence of corneal ulcer, corneal distortion or previous refractive surgical procedure.

As the ICare is based on a different principle, the relationship between central and peripheral readings could be different from those obtained with other tonometers. The goal of the present study was to determine the statistical correlations between central and peripheral IOP readings in order to elucidate if reliable peripheral readings can be obtained in normal corneas with the ICare ${ }^{\circledR}$ rebound tonometer.

\section{Materials and methods}

One-hundred and fifty-three patients (65 males, 88 females), with ages ranging from 21 to 85 years (mean \pm S.D., $55.5 \pm 15.2$ ), were selected to participate in this study.

Inclusion criteria required that the subjects did not suffer from any ocular condition or injury at the time of the study. None of the subjects exhibited corneal pathology or corneal scarring, or had previously undergone corneal surgery. All procedures followed the guidelines of the Declaration of Helsinki and were approved by the Scientific Committee of the School of Sciences at University of Minho (Portugal).

Only the values obtained for the right eye were considered for statistical purposes. IOP was assessed using the ICare ${ }^{\circledR}$ rebound tonometer (Tiolat Oy). After explaining the nature of the experimental procedures, informed consent was obtained from each subject prior to data acquisition. IOP was assessed with the ICare $^{\circledR}$ after an ocular health assessment with slit lamp, and fundus examination through direct ophthalmoscopy. The measurements were carried out by a trained clinician, following the recommendations of the manufacturer as described earlier (Fernandes et al., 2005). Three valid series were taken at centre, nasal and temporal locations. Measurements at the three locations were randomly performed in order to minimise the potential effect of first readings on subsequent measurements. Peripheral measurements were taken at a constant distance of about $2 \mathrm{~mm}$ from the limbus in the nasal and temporal regions of the horizontal meridian. For each peripheral measurement, the patient was asked to look at a peripheral fixation target to the right and left sides in front of him/her in order to take nasal and temporal readings (only right eye was measured). The distance of each lateral fixation target was placed in order to produce a displacement of the corneal apex of approximately $4 \mathrm{~mm}$ after eye rotation. After rotation, the ICare position was slightly adjusted to ensure that the impact point was approximately $2 \mathrm{~mm}$ from limbus; the thickness of the round tip (about $1.4 \mathrm{~mm}$ ) was used as a reference.

Data were analysed using the statistical package SPSS version 14.0 (SPSS Inc., Chicago, IL, USA). Correlations between central and peripheral measurements were assessed statistically as the mean of the differences compared with zero. The $95 \%$ limits of agreement $(\mathrm{LoA}=$ mean of the difference $\pm 1.96 \times$ S.D. of the differences) were also calculated (Bland and Altman, 1986). The bias was assessed statistically as the mean of the differences compared with zero. The hypothesis of zero bias was examined by ANOVA with Bonferroni post $h o c$ correction of $p$-values. One of the benefits of the trend lines in this analysis is the possibility to check if the bias (mean difference) changes significantly as the average mean value for the given parameter increases or decreases. In summary, the trend lines allow us to verify if the bias depends on the measured value or is consistent across a range of values. The level of significance was established at $\alpha=0.05$. Normal distribution of variables was assessed by KolmogorovSmirnov normality test.

\section{Results}

Table 1 presents the mean values of IOP obtained at central, nasal and temporal peripheral locations. No significant differences were observed between males and females regarding central, nasal or temporal values of IOP with the ICare (Mann-Whitney, $p>0.05$ ). Central IOP readings displayed the highest IOP followed by 
Table 1. Descriptive statistics (mean \pm S.D.) of central and peripheral intraocular pressure (IOP) measured with ICare ${ }^{\circledR}$ tonometer, statistical comparisons and correlation analysis for each pair of readings taken at the three corneal locations

\begin{tabular}{|c|c|c|c|c|c|}
\hline Comparisons & Mean \pm S.D. (range) & \multicolumn{2}{|l|}{ ANOVA } & \multicolumn{2}{|l|}{ Correlation $^{\dagger}$} \\
\hline ICare ${ }^{\circledR}$ central & $14.9 \pm 2.8(10-25)$ & $0.75 \pm 1.18$ & 0.045 & 0.905 & $<0.001$ \\
\hline ICare ${ }^{\circledR}$ central & $14.9 \pm 2.8(10-25)$ & $0.37 \pm 1.36$ & 0.680 & 0.879 & $<0.001$ \\
\hline ICare ${ }^{\circledR}$ temporal & $14.5 \pm 2.7(10-23)$ & & & & \\
\hline ICare ${ }^{\circledR}$ nasal & $14.1 \pm 2.5(9-23)$ & $-0.38 \pm 1.24$ & 0.656 & 0.892 & $<0.001$ \\
\hline
\end{tabular}

Values are in $\mathrm{mmHg}$.

${ }^{\dagger}$ Correlation is significant at the 0.01 level (two-tailed).

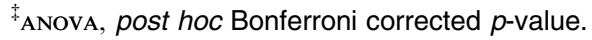

temporal and nasal readings. The lowest IOP values recorded in the nasal location were also associated with a lower standard deviation. The three measurements displayed a high correlation (Table 1), with central vs nasal readings being the most highly correlated $\left(r^{2}=0.905 ; p<0.001\right)$. According to this finding, more than $90 \%$ of the variability in nasal peripheral readings were expected to be explained by the variability in the central readings. Figure 1 illustrates the frequency distribution of differences (in $\mathrm{mmHg}$ ) for nasal and temporal readings against central IOP readings. Almost $80 \%(78.4 \%)$ of patients presented nasal IOP values within $\pm 1 \mathrm{mmHg}$ difference against the central measurement and $73.2 \%$ showed temporal-central differences within this limit. When peripheral readings were compared with each other, $82.3 \%$ of patients displayed nasal and temporal measurements within $\pm 1 \mathrm{mmHg}$.

ANOVA analysis with post hoc Bonferroni correction was applied to evaluate differences between central and peripheral readings and results are presented in Table 1 .

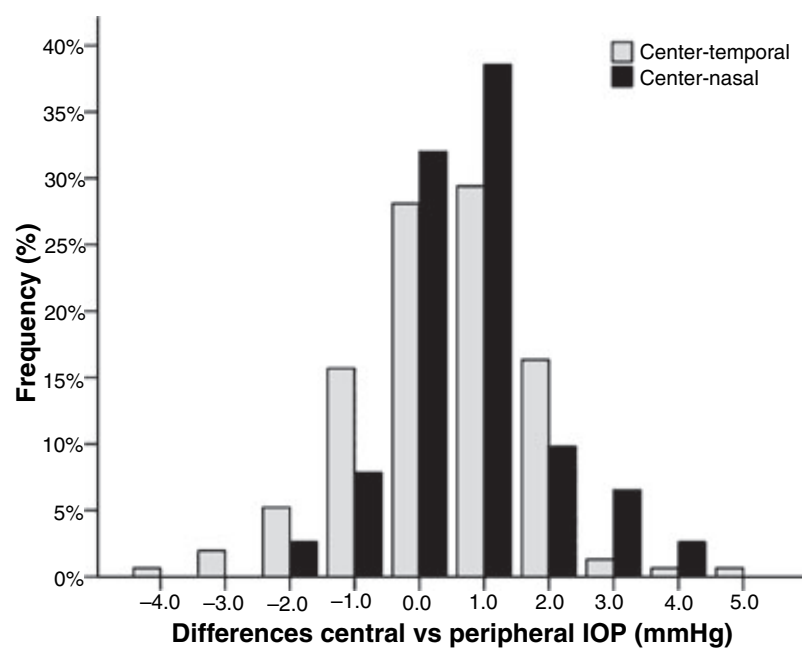

Figure 1. Frequency distribution of differences between central and peripheral intraocular pressure measurements as obtained with ICare ${ }^{\circledR}$ for nasal and temporal locations.
The differences between central and nasal readings were statistically significant $(p=0.045)$, with a mean difference of $0.75 \mathrm{mmHg}$. Conversely, temporal readings did not differ significantly from those recorded at centre with a mean difference of $0.37 \mathrm{mmHg}$.

Figure 2 shows the plots of difference vs mean between central and nasal (Figure 2a), central and temporal (Figure $2 b$ ), and nasal and temporal readings (Figure 2c), respectively. Despite a larger average difference, nasal IOP values were in closer agreement with central IOP than temporal ones as shown by the narrower $95 \%$ confidence interval. No significant trends were observed in differences between measurements as a function of their mean value as seen in Figures $2 a, 2 b$ and $2 c$ except for the comparison between central and nasal readings $(r=0.221 ; p=0.006)$, which display a significant trend towards higher readings at the centre for higher values of IOP and higher readings at the nasal region for lower values. Range between upper and lower limits of agreement is $4.63,5.33$ and $4.86 \mathrm{mmHg}$ for the comparisons presented in Figures $2 a, 2 b$ and $2 c$, respectively. According to this, nasal readings are in closer agreement with central ones, while the least mean difference was found between temporal and central readings (see Table 1).

\section{Discussion}

Limitations to obtaining reliable IOP values in patients at risk of glaucoma will not be uncommon in the future as a consequence of the increasing number of refractive surgery procedures performed in the last two decades. After reaching the age of 40, the increased prevalence of glaucoma creates the need to regularly check IOP in such patients and this requires reliable methods to obtain rapid IOP values, preferably in a non-invasive way. However, with the increasing evidence that altered corneal thickness and curvature will have a negative impact on IOP readings accuracy (Abbasoglu et al., 1998; Montes-Mico and Charman, 2001), new devices 
(a)

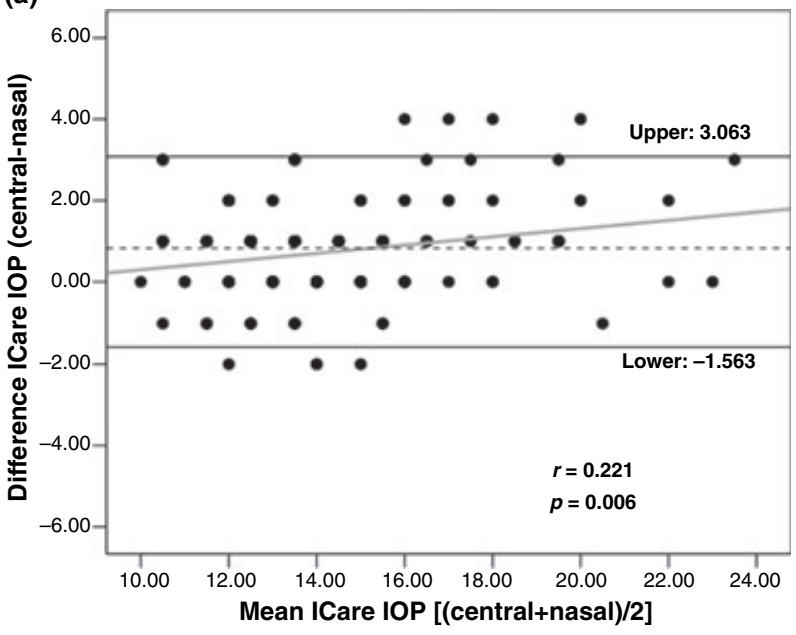

(b)
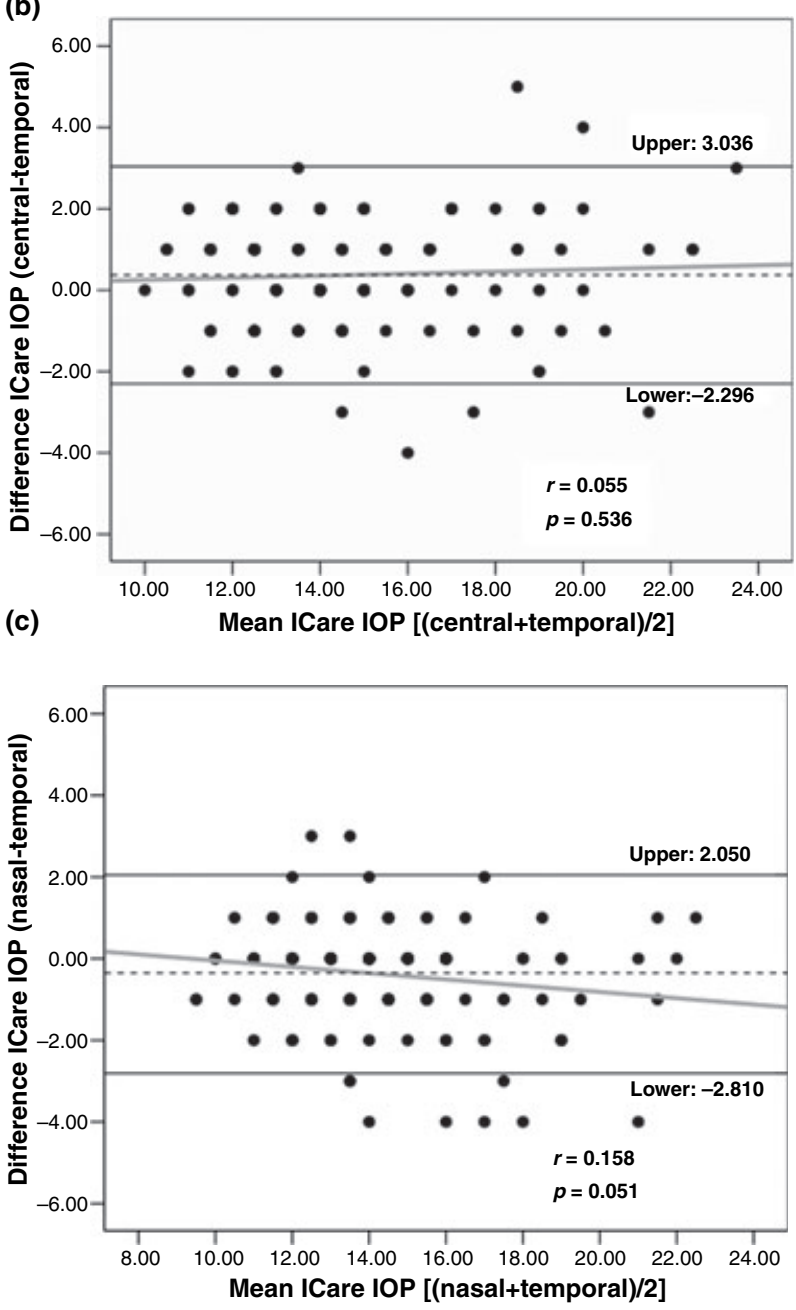

Figure 2. Bland and Altman plots of difference vs mean of intraocular pressure values for (a) central vs nasal, (b) central vs temporal and (c) nasal vs temporal measurements. The mean of the difference (dashed line) and the 95\% confidence interval limits (solid lines and values) are displayed in the graphics. have been launched on to the marketplace like the dynamic contour tonometer (Kaufmann et al., 2003, 2004) and Ocular Response Analyzer (Luce, 2005; Congdon et al., 2006).

Amaral et al. (2006) found that IOP values obtained in the corneal periphery with Tonopen were significantly higher than those taken at corneal centre and this difference is suggested to be due to the increased corneal thickness in the corneal periphery. This was supported by the study of Mok et al. (1999) but not by SullivanMee and Pham (2004), who found no significant differences between central and mid-peripheral readings, despite a positive correlation with corneal thickness being found at both locations. Similarly, other authors did not find statistically significant differences between central and peripheral IOP with GAT in non-operated corneas (Schipper et al., 2000).

Considering the anatomical structure of the normal cornea, and the previous results from Tonopen (Schipper et al., 2000; Sullivan-Mee and Pham, 2004) and GAT (Schipper et al., 2000), we could expect that ICare will give higher values of IOP in the periphery compared with the centre. However, contrary to our initial beliefs, peripheral IOP values obtained in normal corneas with the ICare ${ }^{\circledR}$ were lower than central measurements. This is also contrary to the findings of Martinez-de-la-Casa et al. (2005) who found a correlation between ICare tonometry and corneal thickness. The same authors found in a recent study a positive and significant correlation between central corneal thickness and IOP obtained with rebound tonometry similar to that found for GAT (Martinez-de-la-Casa et al., 2006a). However, the behaviour detected in our study was already reported in a previously published work where changes in central to peripheral IOP relationships showed a relationship with age (Gonzalez-Meijome et al., 2006).

Considering this information, it seems that despite IOP measured with a rebound tonometry correlating significantly with central corneal thickness, the expected increase in IOP is not apparent when measured with a rebound tonometry at corneal peripheral locations despite the cornea being thicker in these regions in normal eyes. We can only speculate that some anatomical facts could be responsible for this behaviour. For example, a less compacted peripheral corneal tissue could be thicker but softer at the same time, thus offering less resistance to the impact tonometer probe than that from the more compacted corneal tissue at centre. This is supported by the results from Boote et al. (2003), who found a mean collagen inter-fibrilar separation $5-7 \%$ larger in the periphery compared with the central $3 \mathrm{~mm}$ of the cornea. The way in which corneal hydration and its differences across the cornea affect inter-fibrilar separation is currently unknown. However it seems reasonable to speculate that if inter-fibrilar 
space increases from centre to periphery without an increase in the number of fibrils or their diameter (Boote et al., 2003) then an increase in the hydration of the peripheral cornea is expected in order to fill the larger gaps between collagen fibrils. As a result, the peripheral cornea will offer less resistance to the impact of the rebound tonometer tip probe giving lower IOP values, as we have found in the present study, instead of higher values that could be expected considering solely the increased peripheral thickness.

In the present study we have observed a high correlation between central and peripheral IOP taken with the ICare rebound tonometer. Small differences between central and peripheral IOP could mean that the instrument variability masks the potential differences that could be present because of the change in the location of the measurement. Davies et al. (2006) gave repeatability data for the ICare ( $\mathrm{LoA} \pm 5.11 \mathrm{mmHg}$ ), although our previous results suggest lower LoA values (about \pm 4.50 and $\pm 4.00 \mathrm{mmHg}$ against Perkins and Goldmann tonometer, respectively). Even in this case, variations in IOP of $<1 \mathrm{mmHg}$ are well within the noise of measurement and could possibly be a result of instrument repeatability masking any potential difference between central and peripheral readings. However the high correlation between central and peripheral readings argue against this hypothesis.

Moreover, peripheral readings would be expected to increase from centre to periphery because of the anatomical thickening of the normal cornea beyond the centre. In fact, the differences are very low or nonexistent, or are opposite to those expected (lower values at periphery than at centre) as seen in Figure 1.

In summary, we have found in the present study that in normal healthy eyes peripheral readings taken with the ICare ${ }^{\circledR}$ rebound tonometer were in high agreement with central ones. While temporal readings have been widely suggested in the literature as an alternative to central ones our results show that with the ICare, both nasal and temporal IOP readings can be used as an alternative to central readings. In a high proportion of patients differences between central and peripheral readings were within an interval of $\pm 1 \mathrm{mmHg}$. These results apply to normal subjects. Further work should be carried out with operated or scarred corneas to verify if similar behaviour is present.

\section{Acknowledgements}

The authors wish to thank Óptica Queirós Lda., where the study was carried out; José Cotta EMS for the loan of the ICare ${ }^{\circledR}$, Ms Sofia Matos, who assisted in the preparation of patients and data acquisition; and Alejandro Cerviño, for assisting with the completion of the manuscript. None of the authors has a commer- cial or financial interest in the instruments presented here.

\section{References}

Abbasoglu, O. E., Bowman, R. W., Cavanagh, H. D. and McCulley, J. P. (1998) Reliability of intraocular pressure measurements after myopic excimer photorefractive keratectomy. Ophthalmology 105, 2193-2196.

Amaral, W. O., Teixeira, R. M., Alencar, L. M., Cronemberger, S. and Calixto, N. (2006) Central and peripheral corneal thickness: influence on the iop measurement by Tonopen. Arq Bras. Oftalmol. 69, 41-45.

Bland, J. M. and Altman, D. G. (1986) Statistical methods for assessing agreement between two methods of clinical measurement. Lancet 1, 307-310.

Boote, C., Dennis, S., Newton, R. H., Puri, H. and Meek, K. M. (2003) Collagen fibrils appear more closely packed in the prepupillary cornea: optical and biomechanical implications. Invest. Ophthalmol. Vis. Sci 44, 2941-2948.

Congdon, N. G., Broman, A. T., Bandeen-Roche, K., Grover, D. and Quigley, H. A. (2006) Central corneal thickness and corneal hysteresis associated with glaucoma damage. Am. J. Ophthalmol. 141, 868-875.

Davies, L. N., Bartlett, H., Mallen, E. A. and Wolffsohn, J. S. (2006) Clinical evaluation of rebound tonometer. Acta Ophthalmol. Scand. 84, 206-209.

Fernandes, P., Diaz-Rey, J. A., Queiros, A., GonzalezMeijome, J. M. and Jorge, J. (2005) Comparison of the ICare $(\mathrm{R})$ rebound tonometer with the Goldmann tonometer in a normal population. Ophthalmic Physiol. Opt. 25, 436-440.

Garcia-Resua, C., Gonzalez-Meijome, J. M., Gilino, J. and Yebra-Pimentel, E. (2006) Accuracy of the new ICare rebound tonometer vs. other portable tonometers in healthy eyes. Optom Vis. Sci 83, 102-107.

Gonzalez-Meijome, J. M., Jorge, J., Queiros, A., Fernandes, P., Montes-Mico, R., Almeida, J. B. and Parafita, M. A. (2006) Age differences in central and peripheral intraocular pressure using a rebound tonometer. Br. J. Ophthalmol. 90, 1495-1500.

Iliev, M. E., Goldblum, D., Katsoulis, K., Amstutz, C. and Frueh, B. (2006) Comparison of rebound tonometry with Goldmann applanation tonometry and correlation with central corneal thickness. Br. J. Ophthalmol 90, 833-835.

van der Jagt, L. H. and Jansonius, N. M. (2005) Three portable tonometers, the TGDc-01, the ICARE and the Tonopen XL, compared with each other and with Goldmann applanation tonometry*. Ophthalmic Physiol. Opt. 25, 429-435.

Jorge, J., Diaz-Rey, J. A., Gonzalez-Meijome, J. M., Almeida, J. B. and Parafita, M. A. (2002) Clinical performance of the Reichert AT550: a new non-contact tonometer. Ophthalmic Physiol. Opt. 22, 560-564.

Jorge, J., Gonzalez-Meijome, J. M., Diaz-Rey, J. A., Almeida, J. B., Ribeiro, P. and Parafita, M. A. (2003) Clinical performance of non-contact tonometry by Reichert AT550 in glaucomatous patients. Ophthalmic Physiol. Opt. 23, 503506. 
Kaufmann, C., Bachmann, L. M. and Thiel, M. A. (2003) Intraocular pressure measurements using dynamic contour tonometry after laser in situ keratomileusis. Invest. Ophthalmol. Vis. Sci. 44, 3790-3794.

Kaufmann, C., Bachmann, L. M. and Thiel, M. A. (2004) Comparison of dynamic contour tonometry with goldmann applanation tonometry. Invest. Ophthalmol. Vis. Sci. 45, 3118-3121.

Kontiola, A. I. (2000) A new induction-based impact method for measuring intraocular pressure. Acta Ophthalmol. Scand. 78, 142-145.

Kontiola, A. and Puska, P. (2004) Measuring intraocular pressure with the Pulsair 3000 and Rebound tonometers in elderly patients without an anesthetic. Graefes Arch. Clin. Exp. Ophthalmol. 242, 3-7.

Kontiola, A. I., Goldblum, D., Mittag, T. and Danias, J. (2001) The induction/impact tonometer: a new instrument to measure intraocular pressure in the rat. Exp. Eye Res. 73, 781-785.

Luce, D. A. (2005) Determining in vivo biomechanical properties of the cornea with an ocular response analyzer. J. Cataract Refract. Surg. 31, 156-162.

Martinez-de-la-Casa, J. M., Garcia-Feijoo, J., Castillo, A. and Garcia-Sanchez, J. (2005) Reproducibility and clinical evaluation of rebound tonometry. Invest. Ophthalmol. Vis. Sci. 46, 4578-4580.

Martinez-de-la-Casa, J. M., Garcia-Feijoo, J., Vico, E., Fernandez-Vidal, A., Benitez Del Castillo, J. M., Wasfi, M. and Garcia-Sanchez, J. (2006a) Effect of Corneal
Thickness on Dynamic Contour, Rebound, and Goldmann Tonometry. Ophthalmology 113, 2162.

Martinez-de-la-Casa, J. M., Garcia-Feijoo, J., Vico, E., Fernandez-Vidal, A., itez del Castillo, J. M., Wasfi, M. and Garcia-Sanchez, J. (2006b) Effect of corneal thickness on dynamic contour, rebound, and goldmann tonometry. Ophthalmology 113, 2156-2162.

Mok, K. H., Wong, C. S. and Lee, V. W. (1999) Tono-Pen tonometer and corneal thickness. Eye 13(Pt 1), 35-37.

Montes-Mico, R. and Charman, W. N. (2001) Intraocular pressure after excimer laser myopic refractive surgery. Ophthalmic Physiol. Opt. 21, 228-235.

Queiros, A., Gonzalez-Meijome, J. M., Fernandes, P., Jorge, J., Almeida, J. B. and Parafita, M. A. (2006) Non-contact tonometry synchronized with cardiac rhythm and its relationship with blood pressure. Ophthalmic Physiol. Opt. 26, 384-391.

Schipper, I., Senn, P., Oyo-Szerenyi, K. and Peter, R. (2000) Central and peripheral pressure measurements with the Goldmann tonometer and Tono-Pen after photorefractive keratectomy for myopia. J. Cataract Refract. Surg. 26, 929 933.

Sullivan-Mee, M. and Pham, F. (2004) Correspondence of Tono-Pen intraocular pressure measurements performed at the central cornea and mid-peripheral cornea. Optometry $\mathbf{7 5}$, 26-32. 